\title{
Ovarian Features after 2 Weeks, 3 Weeks and 4 Weeks Transdermal Testosterone Gel Treatment and Their Associated Effect on IVF Outcomes in Poor Responders
}

\author{
${ }^{\dagger}$ Chung-Hoon Kim ${ }^{1}$, Jun-Woo Ahn ${ }^{2}$, Jei-Won Moon ${ }^{1}$, Sung-Hoon Kim ${ }^{1}$, Hee-Dong Chae ${ }^{1}$ \\ and Byung-Moon Kang ${ }^{1}$ \\ ${ }^{I}$ Division of Reproductive Endocrinology and Infertility, Department of Obstetrics and Gynecology, \\ College of Medicine, University of Ulsan, Asan Medical Center, Seoul 138-736, Korea \\ ${ }^{2}$ Division of Reproductive Endocrinology and Infertility, Department of Obstetrics and Gynecology, \\ College of Medicine, University of Ulsan, Ulsan University Hospital, Ulsan 682-714, Korea
}

\begin{abstract}
This study was performed to investigate the effect of of transdermal testosterone gel (TTG) on controlled ovarian stimulation (COS) and IVF outcomes and ovarian morphology according to pretreatment duration in poor responders. A total of 120 women were recruited for this pilot study. They were randomized into control, 2 weeks, 3 weeks or 4 weeks TTG treatment groups. For three TTG treatment groups, $12.5 \mathrm{mg}$ TTG was applied daily for 2 weeks, 3 weeks or 4 weeks in preceding period of study stimulation cycle. After 3 weeks of TTG pretreatment, significant increase of antral follicle count (AFC) and significant decreases of mean follicular diameter (MFD) and resistance index (RI) value of ovarian stromal artery were observed ( $p=0.026, p<0.001, p<0.01$, respectively). The total dose of $\mathrm{hhFSH}$ administered for COS significantly decreased after 3 and 4 weeks TTG treatment both compared with control group $(p<0.001, p<0.001)$. The numbers of oocytes retrieved and mature oocytes were significanty higher in 3 and 4 weeks TTG treatment groups than control group ( $p<0.001$, $p<0.001$ in the number of oocytes retrieved; $p<0.001, p<0.001$ in the number of mature oocytes). The clinical pregnancy rate and live birth rate were increased only in 4 weeks TTG treatment group compared with control group ( $p=0.030$ and $p=0.042$, respectively). These data demonstrated that TTG pretreatment for 3 to 4 weeks increases AFC and ovarian stromal blood flow, thereby potentially improving the ovarian response to COS and IVF outcome in poor responders undergoing IVF/ICSI.
\end{abstract}

Key words : Transdermal testosterone gel, COS, IVF, Poor responders

\section{INTRODUCTION}

Androgen leads follicular atresia in murine experimental models (Fabregues et al., 2009). However, in primates, androgens had positive effects about follicular development and some synergistic effects with follicle stimulating hormone
(FSH). Androgen supplementation such as testosterone or dihydrotestosterone recruited more growing follicles, and increased granulosa and theca cell proliferation (Vendola et al., 1998). Furthermore, androgen makes follicular FSH receptor expression in granulosa cells (Weil et al., 1999). These androgen actions showed some helpful outcomes

\footnotetext{
Manuscript received 15 July 2014, Received in revised form 28 July 2014, Accepted 4 August 2014

$\dagger$ Corresponding Author : Chung-Hoon Kim, Division of Reproductive Endocrinology and Infertility, Department of Obstetrics and Gynecology, College of Medicine, University of Ulsan, Asan Medical Center, 88, Olympic-ro 43-gil, Songpa-gu, Seoul 138-736, Korea. Tel.: +82-2-3010-3639, Fax: +82-23010-6944, E-mail: chnkim@amc.seoul.kr

This is an Open Access article distributed under the terms of the Creative Commons Attribution Non-Commercial License (http:// creativecommons.org/licenses/by-nc/3.0) which permits unrestricted non-commercial use, distribution, and reproduction in any medium, provided the original work is properly cited.
} 
during in vitro fertilization (IVF) treatment especially for poor ovarian responders (Fabregues et al., 2009; Barad \& Gleicher, 2006).

Poor ovarian response to ovarian stimulation usually indicates a reduced follicular response during assisted reproduction. According to a consensus developed by The European Society for Human Reproduction and Embryology, less than 3 oocytes could be retrieved with a conventional stimulation protocol in poor responders by definition (Ferraretti et al., 2011). So a variety of stimulation protocols have been used, however none of them have been so effective for poor ovarian response. Androgen supplementation protocol is one of them (Gonzalez-Comadran et al., 2012), which have been investigated upon data from experimental animals described above and speculation from women with poly-cystic ovarian syndrome (Massin et al., 2006).

In human data, there was controversy about androgen priming during assisted reproduction. The oral dehydroepiandrosterone (DHEA) supplementation in women with diminished ovarian reserve showed increased ovarian responsiveness and demonstrated significant improved IVF outcomes (Barad \& Gleicher, 2006; Casson et al., 2000). Transdermal testosterone pretreatment also showed increased ovarian sensitivity to gonadotropin (Fabregues et al., 2009).

However, Massin et al. could not demonstrate any beneficial effect on ovarian response to gonadotropin depending on testosterone application. They proposed several reasons about the absence of any beneficial effects of testosterone application, and timing and duration of androgen supplementation were thought to be critical in adequate follicle stimulation and total amount of androgen supplementation was also considered to be an important factor (Massin et al., 2006).

Therefore we conducted a pilot study to investigate the effect of transdermal testosterone gel (TTG) among subgroups classified by use duration in patients who had experienced a poor ovarian response to prior IVF cycles. We observed and compared ovarian morphologies of each groups including antral follicle count, follicular diameter and intra-ovarian blood flow after TTG treatment.

\section{MATERIAL AND METHODS}

\section{Patients}

The study population consisted of total 120 women, and they had been showed poor ovarian responses during prior failed IVF cycles. In detail, women were enrolled who failed to produce over 3 follicles with a mean diameter of $\geq 16 \mathrm{~mm}$, and then less than 3 follicle were retrieved even a high total dose of recombinant human follicle stimulating hormone(rhFSH, > 2,500 IU). All the patients were in good health within normal limits of thyroid, kidney and hepatic laboratory results, and they had regular menstruation period with duration 21-35 days. None of them had ever taken any infertility medication over past 3 months. Our pilot study was performed at a university-based infertility clinic at the Asan Medical Center, Seoul, South Korea. The study population consisted of 120 poor responders who had undergone $120 \mathrm{IVF} / \mathrm{ICSI}$ cycles between Jan 2006 and Jan 2010. Patients were randomly allocated into three TTG treatment groups (TTG use for 2 weeks, 3 weeks or 4 weeks) ( $n=30$ in each groups) and one control group $(n=30)$ by the use of sealed envelopes and a computer-generated list. The sequence of allocation to the four groups was provided to the investigating physicians and randomization was performed as planned according to the randomization list order.

\section{Ovarian stimulation protocol}

Before starting COS cycle, all of the patients had taken estrogen and progesterone pretreatment for 25 days using E2 valerate (Progynova; Bayer Schering Pharma, Berlin, Germany) $1 \mathrm{mg} / \mathrm{d}$ and norethindrone (Primolut; Bayer Schering Pharma) $5 \mathrm{mg} / \mathrm{d}$. In all subgroups, GnRH antagonist multiple- 
dose protocol (MDP) was used for ovarian stimulation. Briefly, 5 days after stopping hormonal pretreatment, rhFSH (Gonal-F; Merck Serono, Geneva, Switzerland) 300 IU/d was started when establishing ovarian and uterine quiescence by using transvaginal ultrasonography. The dose adjustment was based on an individual ovarian response assessed by serial transvaginal ultrasonography every 3 or 4 days. GnRH antagonist cetrorelix (Cetrotide; Merck Serono) $0.25 \mathrm{mg} / \mathrm{d}$ was started when the leading follicle is 13 to 14 $\mathrm{mm}$ in diameter and maintained daily till the day of recombinant hCG (rhCG; Ovidrel; Merck Serono) $250 \mathrm{mg}$ administration. The criteria to induce follicular maturation was the presence of one or more follicle $\geq 18 \mathrm{~mm}$.

Oocyte aspiration was performed under transvaginal ultrasound guidance 35-36 hours after hCG injection, and up to four embryos per patients, after IVF or ICSI, were replaced on the third day after oocyte aspiration. Luteal phase was supported by vaginal progesterone gel $(90 \mathrm{mg} /$ day, Crinone gel $8 \%$; Merck Serono) once daily from the day of oocyte aspiration. Pregnancy diagnosis was made by rising serum b-hCG concentrations and ultrasonographic evidence of a gestational sac. Measurement of b-hCG was performed by radioimmunoassay using an hCG MAIAclone kit (Serono Diagnostics, Woking, U.K.); interassay and intraassay variances were $<10 \%$ and $<5 \%$, respectively.

\section{TTG pretreatment}

For three TTG pretreatment subgroups, $12.5 \mathrm{mg}$ TTG (Testo gel 1\%; Laboratories Besins International, Paris, France) with a $1.25 \mathrm{mg} / \mathrm{d}$ nominal delivery rate of testosterone was applied once daily in the morning, which was administered by the patient onto clean dry healthy skin over both upper arms. This testosterone gel formulation is a hydroalcoholic gel containing $1 \%$ testosterone, and approximately $9-14 \%$ of the steroid in the gel is available in the body (Wang et al., 2004). The dose of TTG, 12.5 $\mathrm{mg} / \mathrm{d}$ was determined on the basis of experimental data in primates (Vendola et al., 1998; Vendola et al., 1999). Once gel applied, patients were instructed to avoid washing the application sites, for 5 hours, but washed their hands thoroughly with soap and water. The duration of TTG pretreatment was varied among the study subgroups, which were 2, 3 and 4 weeks respectively in prece-ding period of study stimulation cycle. Ovarian stimulation was started next day of last testosterone gel application.

\section{Outcome measures}

The primary end point was the total number of mature retrieved oocytes. The secondary endpoints were the number of growing follicles, numbers of fertilized oocytes and good-quality embryos. We also evaluated and analyzed total dose and days of rhFSH administered, implantation rate, clinical pregnancy rate per cycle, and live birth rate per cycle. Clinical pregnancy was indicated in case of the presence of a gestational sac by ultrasonography, and live birth was indicated in case of the delivery of a fetus with signs of life after 20 completed weeks of gestational age. Miscarriage rate per clinical pregnancy was defined as the proportion of patients who failed to continue development before 20 weeks of gestation in all clinical pregnancies.

\section{Statistical analysis}

Results were presented as the mean \pm standard deviation (SD) with $95 \%$ confidence interval. All statistical analysis was performed using SPSS statistical package for Windows, version 11.0 (SPSS Inc, Chicago, IL). Analysis of variance (ANOVA) was performed to compare the mean values among four groups. Chi-square test, and Fisher's exact test were performed for the comparisons of fraction, where applicable. $P<0.05$ was considered to be obtained statistical significance.

\section{RESULTS}

As presented in Table 1, patients' characteristics including 
Table 1. Patient characteristics

\begin{tabular}{|c|c|c|c|c|c|}
\hline & Control & $\begin{array}{c}2 \mathrm{wks} \\
\text { treatment }\end{array}$ & $\begin{array}{c}3 \mathrm{wks} \\
\text { treatment }\end{array}$ & $\begin{array}{c}4 \text { wks } \\
\text { treatment }\end{array}$ & $P$ \\
\hline No. of patients & 30 & 30 & 30 & 30 & \\
\hline Age of patients (yrs) & $37.6 \pm 3.4$ & $37.9 \pm 2.9$ & $38.3 \pm 3.1$ & $38.1 \pm 3.0$ & NS \\
\hline Age of husbands (yrs) & $43.3 \pm 4.3$ & $42.9 \pm 3.3$ & $43.5 \pm 4.2$ & $43.0 \pm 3.6$ & NS \\
\hline Infertility duration (mon) & $51.8 \pm 25.8$ & $49.7 \pm 23.9$ & $55.2 \pm 33.4$ & $53.3 \pm 28.5$ & NS \\
\hline BMI $\left(\mathrm{kg} / \mathrm{m}^{2}\right)$ & $21.6 \pm 2.4$ & $21.4 \pm 2.1$ & $21.8 \pm 2.3$ & $21.6 \pm 2.2$ & NS \\
\hline No. of nullipara (\%) & $60.0(18 / 30)$ & $63.3(19 / 30)$ & $56.7(17 / 30)$ & $66.6(20 / 30)$ & NS \\
\hline No. of patients with $\mathrm{AFC} \leq 5$ & $90.0(27 / 30)$ & $90.0(27 / 30)$ & $96.7(29 / 30)$ & $86.7(26 / 30)$ & NS \\
\hline $\mathrm{AFC}$ & $4.7 \pm 0.8$ & $4.6 \pm 0.7$ & $4.4 \pm 0.6$ & $4.8 \pm 1.0$ & NS \\
\hline Basal FSH (IU/L) & $8.7 \pm 1.2$ & $8.5 \pm 0.8$ & $8.9 \pm 1.1$ & $8.8 \pm 1.1$ & NS \\
\hline
\end{tabular}

Values are means \pm SDs. $\mathrm{BMI}=$ body mass index; $\mathrm{AFC}=$ antral follicle count; $\mathrm{FSH}=$ follicle stimulating hormone; $\mathrm{NS}=$ not significant

age, duration of infertility, body mass index (BMI), basal $\mathrm{FSH}$, and antral follicle count (AFC) were similar in four groups. Each three TTG pretreatment and control groups consisted of 30 cycles initiated, corresponding to 30 patients. Three cycles $(10.0 \%)$ of control group and 2 cycles $(6.7 \%)$ of 2 weeks TTG group were cancelled, because of no follicular development before oocyte aspiration or no oocytes obtained despite a follicular aspiration for oocyte retrieval. Statistical difference was not found in overall cancellation rate among all TTG pretreatment and control groups (Table 2).

Both serum $\mathrm{T}$ and free $\mathrm{T}$ levels were significantly higher in 2 weeks, 3 weeks and 4 weeks TTG treatment groups compared with control group $(p<0.05, p<0.01, p<0.01$, respectively in serum $\mathrm{T}$ level; $p<0.05, p<0.01, p<0.01$ respectively in serum free T level). After 2 weeks of TTG treatment, AFC, mean follicular diameter (MFD) and resistance index (RI) value of ovarian stromal artery (OSA) were similar to those in control group. COS results and IVF outcome did not show any significant differences between 2 weeks TTG treatment and control groups (Table 2). However, TTG pretreatment for 3 weeks resulted in significant increase of AFC and significant decreases of MFD and RI value of OSA ( $p=0.026, p<0.001, p<0.01$, respectively) (Table 2). Total dose and days of rhFSH used were significantly fewer in 3 weeks TTG group than those in control group ( $p<0.001, p<0.01$, respectively). The numbers of oocytes retrieved, mature oocytes and fertilized oocytes were significantly higher than in control group $(p<0.001$, $p<0.001, p<0.001)$. There were no significant differences in clinical pregnancy rate (CPR) per cycle initiated and live birth rate between 3 weeks TTG treatment and control groups. After 4 weeks of TTG pretreatment, AFC increased, and MFD and RI value of OSA decreased, compared with control group ( $p=0.002, p<0.001, p<0.001$, respectively). Total dose and days of rhFSH administered were signifycantly fewer than those in control group $(p<0.001$, $p<0.001)$. The numbers of oocytes retrieved, mature oocytes and fertilized oocytes, CPR per cycle initiated and live birth rate were significantly higher in 4 weeks TTG treatment group than those in control group $(p<0.001$, $p<0.001, p<0.001, p=0.030, p=0.042$, respectively) (Table 2). No local or systemic adverse effect according to TTG application was reported. 
Table 2. Comparison of controlled ovarian stimulation results and IVF-ET outcome

\begin{tabular}{lcccc}
\hline \hline & Control & $\begin{array}{c}2 \text { wks } \\
\text { treatment }\end{array}$ & $\begin{array}{c}3 \text { wks } \\
\text { treatment }\end{array}$ & $\begin{array}{c}4 \text { wks } \\
\text { treatment }\end{array}$ \\
\hline No. of cycles initiated & 30 & 30 & 30 & 30 \\
No. of cycles retrieved & 28 & 29 & 30 & 30 \\
No. of ET cycles & 27 & 28 & 30 & 30 \\
No. of cycles cancelled & $3(10.0 \%)$ & $2(6.7 \%)$ & 0 & 0 \\
On stimulation day 1 & & & & \\
AFC & $4.0 \pm 1.3$ & $4.1 \pm 1.2$ & $4.9 \pm 1.1^{\mathrm{a}}$ & $5.2 \pm 1.0^{\mathrm{b}}$ \\
$\quad$ MFD & $5.9 \pm 0.6$ & $5.7 \pm 0.5$ & $5.2 \pm 0.4^{\mathrm{c}}$ & $4.7 \pm 0.4^{\mathrm{c}}$ \\
$\quad$ RI of OSA & $0.95 \pm 0.03$ & $0.94 \pm 0.03$ & $0.92 \pm 0.03^{\mathrm{d}}$ & $0.89 \pm 0.03^{\mathrm{c}}$ \\
Total dose of rhFSH (IU) & $3,025.0 \pm 425.9$ & $2,765.7 \pm 567.8$ & $2,596.7 \pm 335.3^{\mathrm{c}}$ & $2,643.5 \pm 389.0^{\mathrm{c}}$ \\
Days of rhFSH administered & $10.6 \pm 1.5$ & $10.2 \pm 1.7$ & $9.6 \pm 1.1^{\mathrm{c}}$ & $9.8 \pm 1.0^{\mathrm{c}}$ \\
No. of oocytes retrieved & $3.9 \pm 1.3$ & $4.3 \pm 1.6$ & $5.3 \pm 2.0^{\mathrm{c}}$ & $5.8 \pm 1.9^{\mathrm{c}}$ \\
No. of mature oocytes & $3.1 \pm 1.1$ & $3.6 \pm 1.3$ & $4.5 \pm 1.8^{\mathrm{c}}$ & $4.9 \pm 1.6^{\mathrm{c}}$ \\
No. of fertilized oocytes & $3.1 \pm 1.1$ & $3.5 \pm 1.3$ & $4.2 \pm 1.7^{\mathrm{c}}$ & $4.6 \pm 1.6^{\mathrm{c}}$ \\
No. of grade I, II embryos & $1.5 \pm 0.6$ & $1.6 \pm 0.6$ & $2.2 \pm 0.6^{\mathrm{a}}$ & $2.1 \pm 0.7^{\mathrm{a}}$ \\
No. of embryos transferred & $2.9 \pm 0.9$ & $2.7 \pm 0.7$ & $2.9 \pm 0.9$ & $2.9 \pm 0.8$ \\
CPR per cycle initiated (\%) & $10.0(3 / 30)$ & $16.7(5 / 30)$ & $30.0(9 / 30)$ & $36.7(11 / 30)^{\mathrm{e}}$ \\
Miscarriage rate per clinical pregnancy (\%) & $33.3(1 / 3)$ & $20.0(1 / 5)$ & $22.2(2 / 9)$ & $18.2(2 / 11)$ \\
Live birth rate per cycle initiated (\%) & $6.7(2 / 30)$ & $13.4(4 / 30)$ & $20.0(6 / 30)$ & $30.0(9 / 30)^{\mathrm{f}}$ \\
Multiple pregnancy rate (\%) & 0 & 0 & $22.2(2 / 9)$ & $18.2(2 / 11)$ \\
\hline Valy are ma & & & & \\
\hline
\end{tabular}

Values are means \pm standard deviations. ET $=$ embryo transfer; AFC $=$ antral follicle count; MFD $=$ mean follicular diameter; $\mathrm{RI}=$ resistance index; OSA = ovarian stromal artery; $\mathrm{rhFSH}=$ recombinant human follicle stimulating hormone; $\mathrm{CPR}=$ clinical pregnancy rate

${ }^{\mathrm{a}} p<0.050$ vs control group, ${ }^{\mathrm{b}} p=0.002$ vs control group, ${ }^{\mathrm{c}} p<0.001$ vs control group, ${ }^{\mathrm{d}} p<0.010$ vs control group, ${ }^{\mathrm{e}} p=0.030$ vs control group, ${ }^{\mathrm{f}} p=0.042$ vs control group

\section{DISCUSSION}

This pilot study demonstrated the beneficial effect of transdermal testosterone gel treatment on COS and IVF in women with poor ovarian responses during prior failed IVF cycles. Indeed the testosterone effect was occurred at least 3 weeks after application of the gel. The TTG treated patients for 3 to 4 weeks responded to FSH more than control or 2 weeks treated patients with result of more numbers of growing follicles during stimulation and oocytes retrieved. Total dose of consumed gonadotropin decreased, and there were no local or systemic adverse side effects.

The $25 \%$ of circulating testosterone comes from ovary in regularly cycling women, and these levels appeared to decrease with age (Barbieri et al., 2005). Similarly, women with lower ovarian reserve were predicted to have low testosterone levels like aging women, even though it's subclinical. According to testosterone threshold analysis during IVF cycles, higher baseline testosterone level appeared to be associated with successful pregnancy (Frattarelli \& Peterson, 2004). Barbieri et al. reported significant positive 
correlation between serum testosterone and numbers of oocytes retrieved (Barbieri et al., 2005). In this perspective, basal hormonal secretion should be more carefully assessed for poor ovarian responders and they could be affected by testosterone level more than who are not a poor responders during gonadotropin stimulation.

Poor ovarian response was reported as $9-25 \%$ of assisted reproduction (de los Santos et al., 2013). Several strategies have not fulfilled to increase reproductive outcomes for these poor responders, and commonly used approach is to make a high androgen intra-ovarian environment. So, dehydroepiandrosterone (DHEA), transdermal testosterone, aromatase inhibitor or recombinant luteinizing hormone (LH) was commonly administered in clinical practice (de los Santos et al., 2013; Fanchin et al., 2011). The roles of androgens in ovarian folliculogenesis have been proposed, and basically androgens are essential substrates for ovarian steroidogenesis according to 2-cell two- gonadotropin theory (Ryan et al., 1968). Mediated by androgen receptor, testosterone and dihydrotestosterone (DHT) have made preantral and small antral follicular growth significantly in primates' ovaries. Granulosa and theca cell proliferation were significantly increased also in these follicles (Vendola et al., 1998). Weil et al. documented androgen receptor gene expression mostly in granulosa cells was up-regulated and granulosa cell FSH receptor mRNA was increased also by testosterone (Weil et al., 1998). Insulin-like growth factor I (IGF-I) and IGF-I receptor mRNA were markedly increased in primordial follicle oocytes in the testosterone or DHTtreated monkeys (Vendola et al., 1999). They suggested androgens stimulate early, gonadotropin independent stages of follicular growth and enhance responsiveness to gonadotropin (Vendola et al., 1998; Weil et al., 1998).

In the study about human granulosa cells and follicular fluids of small antral follicles, significant correlative mRNA expression was observed between androgen receptor and FSH receptor. Furthermore, follicular fluid testosterone levels were highly significantly correlated with FSH receptor mRNA expression (Nielsen et al., 2011). There were a few studies about testosterone priming for COS, but the results were controversial and limited. Fabregues et al reported increased ovarian follicular responses to gonadotropin stimulation in previous low responder IVF patients. They used transdermal testosterone patch with $2.5 \mathrm{mg} / \mathrm{day}$ nominal delivery rate during 5 days preceding gonadotropin treatment for testosterone priming. However, there was no statistical difference about the numbers of retrieved oocytes and embryos, and the rate of clinical pregnancies (Fabregues et al., 2009). Another prospective, randomized trial performed by Massin et al. failed to demonstrate any significant beneficial effect of testosterone on ovarian response to $\mathrm{FSH}$. In this trial, testosterone transdermal gel was applied for 2 weeks before ovarian stimulation, allowing a daily dosing of $10 \mathrm{mg}$ of testosterone with $10 \%$ of absorption approximately. They restricted the duration of application to prevent any side effects (Massin et al., 2006). The timing and duration of testosterone supplementation, and moreover the total amount of supplementation were presented as critical factors for absence of testosterone priming effect.

In this point, our trial has some strengths and favorable results. Most of all, the study was designed to reveal testosterone effect according to the duration of application. The positive effects to ovarian stimulation were observed and moreover IVF outcomes were improved after 3 to 4 weeks of testosterone gel application. CPR were significantly increased up to $36.7 \%$ especially after 4 weeks TTG treatment. These results could be highlighted because the study population was poor responder. However, data on the dose-response effects of androgens are still limited. In primates study, the threshold effect of androgens on follicular function has been postulated. When high dose of androgens were used for 5 days before and continuing through out FSH and LH treatment, antagonistic actions to 
gonadotropin stimulation were observed (Zeleznik et al., 2004). Furthermore intra-ovarian bioavailability of androgens administered systemically at nonvilrilzing dose needs further investigation (Fanchin et al., 2011). The proper duration of androgen pretreatment should be taken into consideration also. Considering androgens' effect to early stage follicles, pretreatment duration could be taken for several weeks before ovarian stimulation (Weil et al., 1998), but TTG should be applied for the shortest duration with positive effect.

Ovarian morphology in circumstances of androgen excess is well known from patients of polycystic ovarian syndrome (PCOS). It is characterized by excessive AFCs, which have been positively correlated with circulating androgen concentrations (Jonard et al., 2003; Pigny et al., 2003). In case of congenital adrenal hyperplasia or some virilizing tumors, which exposed women to androgen for long duration, comparable ovarian morphologies in PCOS were observed (Fanchin et al., 2011). After high doses testosterone taken by noncastrated female to male transsexuals, multifollicular ovarian pattern like PCOS was observed by some investigators (Futterweit \& Deligdisch, 1986; Pache et al., 1991). In our study, after 3 weeks of TTG treatment, AFCs were significantly increased and MFD was significantly decreased. Because androgen promotes early stages of follicular growth, MFD had been thought to be decreased. The improvement of intra-ovarian blood flow was demonstrated also, and further investigations are required.

In conclusion, TTG pretreatment for 3 to 4 weeks before ovarian stimulation increases $\mathrm{AFC}$ and ovarian stromal blood flow and therefore, potentially can improve the ovarian response to $\mathrm{COS}$ and IVF outcome in poor responders undergoing IVF/ICSI.

\section{REFERENCES}

Barad D, Gleicher N (2006) Effect of dehydroepiandrosterone on oocyte and embryo yields, embryo grade and cell number in IVF. Hum Reprod 21:2845-2849.

Barbieri RL, Sluss PM, Powers RD, McShane PM, Vitonis A, Ginsburg E (2005) Association of body mass index, age, and cigarette smoking with serum testosterone levels in cycling women undergoing in vitro fertilization. Fertil Steril 83:302-308.

Casson PR, Lindsay MS, Pisarska MD, Carson SA, Buster JE (2000) Dehydroepiandrosterone supplementation augments ovarian stimulation in poor responders: a case series. Hum Reprod 15:2129-2132.

de los Santos MJ, Garcia-Laez V, Beltran D, Labarta E, Zuzuarregui JL, Alama P (2013) The follicular hormonal profile in low-responder patients undergoing unstimulated cycles: Is it hypoandrogenic? Hum Reprod 28:224-229. Fabregues F, Penarrubia J, Creus M, Manau D, Casals G, Carmona F (2009) Transdermal testosterone may improve ovarian response to gonadotrophins in low-responder IVF patients: a randomized, clinical trial. Hum Reprod 24:349-359.

Fanchin R, Frydman N, Even M, Berwanger da Silva AL, Grynberg M, Ayoubi JM (2011) Androgens and poor responders: are we ready to take the plunge into clinical therapy? Fertil Steril 96:1062-1065.

Ferraretti AP, La Marca A, Fauser BC, Tarlatzis B, Nargund G, Gianaroli L (2011) ESHRE consensus on the definition of 'poor response' to ovarian stimulation for in vitro fertilization: the Bologna criteria. Hum Reprod 26:1616-1624

Frattarelli JL, Peterson EH (2004) Effect of androgen levels on in vitro fertilization cycles. Fertil Steril 81:1713-1714.

Futterweit W, Deligdisch L (1986) Histopathological effects of exogenously administered testosterone in 19 female to male transsexuals. J Clin Endocrinol Metab 62:1621. 
Gonzalez-Comadran M, Duran M, Sola I, Fabregues F, Carreras R, Checa MA (2012) Effects of transdermal testosterone in poor responders undergoing IVF: systematic review and meta-analysis. Reprod Biomed Online 25:450-459.

Jonard S, Robert Y, Cortet-Rudelli C, Pigny P, Decanter C, Dewailly D (2003) Ultrasound examination of polycystic ovaries: is it worth counting the follicles? Hum Reprod 18:598-603.

Massin N, Cedrin-Durnerin I, Coussieu C, Galey-Fontaine J, Wolf JP, Hugues JN (2006) Effects of transdermal testosterone application on the ovarian response to FSH in poor responders undergoing assisted reproduction technique--a prospective, randomized, double-blind study. Hum Reprod 21:1204-1211.

Nielsen ME, Rasmussen IA, Kristensen SG, Christensen ST, Mollgard K, Wreford AE (2011) In human granulosa cells from small antral follicles, androgen receptor mRNA and androgen levels in follicular fluid correlate with FSH receptor mRNA. Mol Hum Reprod 17:63-70.

Pache TD, Chadha S, Gooren LJ, Hop WC, Jaarsma KW, Dommerholt HB (1991) Ovarian morphology in longterm androgen-treated female to male transsexuals. A human model for the study of polycystic ovarian syndrome? Histopathology 19:445-452.

Pigny P, Merlen E, Robert Y, Cortet-Rudelli C, Decanter C, Jonard S (2003) Elevated serum level of antimullerian hormone in patients with polycystic ovary syndrome: relationship to the ovarian follicle excess and to the follicular arrest. J Clin Endocrinol Metab 88:5957-5962.
Ryan KJ, Petro Z, Kaiser J (1968) Steroid formation by isolated and recombined ovarian granulosa and tehcal cells. J Clin Endocrinol Metab 28:355-358.

Vendola KA, Zhou J, Adesanya OO, Weil SJ, Bondy CA (1998) Androgens stimulate early stages of follicular growth in the primate ovary. J Clin Invest 101:26222629.

Vendola K, Zhou J, Wang J, Famuyiwa OA, Bievre M, Bondy CA (1999) Androgens promote oocyte insulinlike growth factor I expression and initiation of follicle development in the primate ovary. Biol Reprod 61: 353-357.

Wang C, Cunningham G, Dobs A, Iranmanesh A, Matsumoto AM, Snyder PJ (2004) Long-term testosterone gel (AndroGel) treatment maintains beneficial effects on sexual function and mood, lean and fat mass, and bone mineral density in hypogonadal men. J Clin Endocrinol Metab 89:2085-2098.

Weil SJ, Vendola K, Zhou J, Adesanya OO, Wang J, Okafor J (1998) Androgen receptor gene expression in the primate ovary: cellular localization, regulation, and functional correlations. J Clin Endocrinol Metab 83: 2479-2485.

Weil S, Vendola K, Zhou J, Bondy CA (1999) Androgen and follicle-stimulating hormone interactions in primate ovarian follicle development. J Clin Endocrinol Metab 84:2951-2956.

Zeleznik AJ, Little-Ihrig L, Ramasawamy S (2004) Administration of dihydrotestosterone to rhesus monkeys inhibits gonadotropin-stimulated ovarian steroidogenesis. J Clin Endocrinol Metab 89:860-866. 